\title{
IMPACTO DE ESTRATEGIAS PARA AUMENTAR EL CONSUMO DE FRUTAS Y VERDURAS EN COLOMBIA
}

\section{IMPACT OF STRATEGIES TO INCREASE THE CONSUMPTION OF FRUITS AND VEGETABLES IN COLOMBIA}

\author{
Gloria Esperanza Prada G. (1, 2), Oscar Fernando Herrán F. $(1,2)$
}

(1) Escuela de Nutrición y Dietética. Universidad Industrial de Santander, Colombia

(2) Grupo de investigación "Observatorio Epidemiológico de Enfermedades Cardiovasculares".

Centro de Investigaciones Epidemiológicas. Universidad Industrial de Santander, Bucaramanga, Colombia.

\begin{abstract}
During the years 2007-2008 a study was carried out in Lebrija (Colombia) to evaluate the impact of two strategies implemented in families with school-age children to increase the consumption of fruits and vegetables at home. Sixteen homes were intervened with the A strategy while twenty three homes were intervened with a $B$ strategy. Thirty four homes served like controls. These strategies included, among others, informative shops, preparation of recipes, activities in group and evaluation of tasks. At the initiation, $3.0 \%$ of the intervened homes consumed five or more portions increasing to $26.3 \%$ at the end of the intervention, an increment of 8.8 times. In the control group the consumption of five or more daily portions was of $2.0 \%$ at the beginning and $0.0 \%$ at the end. There was no difference between strategy $A$ and $B$ in the increase in consumption (0\% to $23.5 \%$ and $4.3 \%$ to $28.6 \%$, respectively. The two evaluated strategies had positive impact, the A on the consumption of fruits and vegetables, the $B$ on the consumption of fruits.
\end{abstract}

Key words: impact evaluation, fruits, vegetables, randomized controlled trials, intervention studies, Colombia.

Este trabajo fue recibido el 20 de Mayo de 2009 y aceptado para ser publicado el 10 de Julio de 2009.

\section{INTRODUCCIÓN}

La Organización Mundial de la Salud (OMS) y la Organización de las Naciones Unidas para la Alimentación y la Agricultura (FAO), lanzaron en 2003 la Estrategia Mundial sobre Régimen Alimentario, Actividad Física y Salud, ésta incluye la iniciativa para aumentar la producción y el consumo de frutas y verduras (FV) (1), una de las recomendaciones es el consumo de por lo menos cinco porciones diarias de FV (2).

Dietas con insuficiente cantidad de frutas y verduras se han asociado con el desarrollo de las enfermedades crónicas no transmisibles (ECNT) (3). A pesar de que la recomendación de consumo diario de FV es de $400 \mathrm{~g}$, en países en vía de desarrollo el consumo medio es inferior a $100 \mathrm{~g}(4,5)$. Según lo reportado por la Encuesta Nacional de la Situación Nutricional 2005 (ENSIN-2005), en Colombia el 35,3\% y el 27,9\% de la población, no incluyen frutas y verduras en su alimentación (6).

Las investigaciones muestran que los aspectos fa- vorecedores del consumo de FV son la disponibilidad de preparaciones que permitan su consumo inmediato, el reconocimiento del efecto positivo en la salud (7), la disponibilidad comercial (7-11), los conocimientos relacionados con FV (10-13), las preferencias y las habilidades relacionadas con la preparación de los alimentos y la planificación del menú (8-9). Específicamente en el caso de las frutas influye el costo, la intolerancia y lo que representa socialmente su consumo, en las verduras, las propiedades organolépticas, el tiempo requerido para su preparación y el conocimiento de recetas $(7,8,14)$.

Para dar respuesta al bajo consumo de FV la OMS plantea la implementación de estrategias educativas, éstas pueden estar orientadas a promocionar hábitos alimentarios saludables o modificarlos; implicando complejos cambios (16), por lo que éstas requirieren de sostenibilidad en el tiempo para evidenciar su impacto.

Las estrategias deben trascender los propósitos en términos de resultados hacia el monitoreo, evaluación y 
difusión (9). La evaluación de impacto es fundamental para la formulación de políticas (17). La OMS incluye como áreas estratégicas para la prevención de enfermedades crónicas la generación de conocimiento con relación a intervenciones evaluadas exitosamente y que integren distintos sectores de la sociedad (15).

En Girón, Colombia durante 2006 se implementó y evaluó una intervención de tipo educativa para aumentar el consumo de FV que mostró resultados positivos en familias con niños escolarizados (18). El objetivo de este estudio fue replicar esta estrategia, e implementar otra intervención fundamentada en ella pero ajustada, para evaluar si haciéndola más viable se obtienen los mismos resultados.

\section{SUJETOS Y MÉTODOS}

Lebrija, es una ciudad pequeña, cuenta con 34000 habitantes, su economía esta ligada al sector agrícola y pecuario, la temperatura media es de $23{ }^{\circ} \mathrm{C}$ y esta situada a 22 kilómetros por carretera pavimentada de Bucaramanga, la ciudad más desarrollada de la región nororiental de Colombia. Durante ocho meses (julio de 2007 a febrero de 2008), se realizó un ensayo comunitario en el que 39 familias fueron intervenidas y 34 sirvieron como control. Dieciséis de las 39 familias fueron intervenidas con la estrategia A, las 23 restantes con la estrategia B.

Tamaño de la muestra. Quince familias por cada grupo; control e intervenido, permiten estimar diferencias en el consumo diario de frutas o verduras de mínimo 0,5 porciones, aceptando una frecuencia de consumo basal para frutas o verduras de una porción diaria, con desviación estándar de 0,8 y tres medidas, una de base y dos de seguimiento; una correlación entre medidas de 0,8 , alfa de 0,05 y poder de 0,90 . Los parámetros que permitieron el cálculo de la muestra fueron obtenidos de estudios realizados sobre el patrón alimentario de población de todos los estratos socioeconómico de la misma área $(19,20)$.

Selección de las familias. Las familias fueron seleccionadas a través de los alumnos de una institución de educación básica primaria. Usando listados de los niños, se asignaron sus familias aleatoriamente a cada una de las dos estrategias. El número de familias seleccionadas fue mayor al requerido para compensar las posibles pérdidas en un seguimiento como el realizado con duración de ocho meses. La alcaldía municipal y las directivas del colegio avalaron y facilitaron la logística para realizar el estudio.

Estrategia de intervención A. Estuvo dirigida a las madres o personas responsables de la alimentación familiar. Después de informarlas detalladamente sobre los aspectos logísticos y las temáticas a desarrollar, se establecieron acuerdos para el desarrollo de las actividades. En un restaurante comunitario se realizó un curso sobre alimentación saludable que incluyó once talleres semanales, con duración de tres horas. Los talleres iniciaban con discusiones sobre alimentación saludable, la relación entre alimentación, salud y enfermedad, características nutricionales de las recetas preparadas, manipulación adecuada de alimentos, planificación del menú y manejo eficiente de la compra de alimentos; seguidamente se preparaba la receta con FV, al finalizar la sesión se entregaba un resumen de lo visto con un ejercicio de aplicación que debía desarrollarse en el hogar y presentarse al inicio del siguiente encuentro. Estas memorias se colectaban en una carpeta que servía como material de consulta para realizar los ejercicios de aplicación en el hogar. Esta intervención incluyó acompañamiento mediante visitas domiciliarias. La culminación del curso incluyó un concurso de recetas, la entrega de certificados de asistencia, libros de recetas y un premio especial para los asistentes que permanecieron hasta el final de la intervención.

Estrategia de intervención B. Con los alumnos se realizaron contactos semanales en el aula de clase y con el grupo familiar, contacto indirecto a través de comunicaciones escritas semanales, llamadas telefónicas y encuentros presenciales en reuniones mensuales. El trabajo con los escolares consistió en sesiones educativas de una hora de duración, éstas se planearon conjuntamente con los docentes y tuvieron como objetivo dar a conocer las características nutricionales de los alimentos y la importancia de una alimentación saludable, se realizaron preparaciones y degustaciones de recetas con FV.

Al igual que en la estrategia A, se entregaba al niño un resumen del tema desarrollado en cada sesión, ejemplos de recetas, y trabajos de aplicación que debía desarrollar con su familia. Estas memorias se colectaban en una carpeta que llevaba a su hogar; incluía además información nutricional para las familias y comunicaciones sobre el avance del proyecto. Se buscaba que el niño fuera un motivador para adoptar una alimentación saludable en el hogar. Con los responsables de la preparación de alimentos, se trataron los mismos temas de la estrategia A, la diferencia consistió, en que la familia recibía la información nutricional mediante una ficha que el niño le entregaba semanalmente. En los encuentros grupales se aclaraban las dudas, se afianzaban los conocimientos y se degustaban las recetas con FV. La culminación de la intervención incluyó la entrega de libros de recetas y un premio especial a los registros más completos -carpeta con todas las tareas-. Esta intervención no incluyó acompañamiento mediante visitas domiciliarias, pero 
si un seguimiento telefónico. En total se realizaron 10 sesiones con los niños y tres con los padres.

El diseño de las estrategias se basó en otra realizada previamente en la región y ajustada según sugerencias de grupos focales, como lo recomiendan los expertos (21). En las dos estrategias fue establecida una línea de base con encuestas sociodemográficas y de consumo de FV. La implementación se realizó con estudiantes de último año de la Escuela de Nutrición y la asesoría de los docentes de prácticas, utilizando como material de apoyo libros de recetas validados en grupos comunitarios $(22,23)$. El grupo investigador y el grupo coordinador se encargó de evaluar el progreso de la intervención de acuerdo a un plan preestablecido.

Proceso de variables $\mathbf{y}$ análisis estadístico. El estrato socioeconómico fue determinado con base en el recibo de la luz. La ingesta de FV se obtuvo de un cuestionario de frecuencia de consumo familiar (CFC), que con base en nueve categorías de frecuencia indagó por el consumo de FV en el último mes. La frecuencia de consumo fue reescalada para obtener la frecuencia de consumo mensual. El CFC incluyó una lista de dieciocho frutas y diecisiete verduras. Un recordatorio del consumo de FV durante las últimas 24 horas $(\mathrm{R} 24 \mathrm{H})$ permitió obtener el número de porciones/día. El número de porciones de FV fue establecido con base a medidas caseras estandarizadas previamente. Para describir las características de las familias estudiadas se utilizaron promedios en las variables continuas y proporciones en las categóricas, con sus intervalos de confianza del $95 \%$ (IC). Para determinar si existían diferencias estadísticamente significantes entre el grupo control e intervenido se aplicaron pruebas $t$ de student y de $\mathrm{Chi}^{2} \mathrm{y}$ el método propuesto por Bland y Alman (24).

Evaluación de impacto. Seis diferentes momentos establecidos en el tiempo y usados para evaluar el impacto de las estrategias se agruparon de la siguiente manera; el promedio de las dos primeras medidas se denominó el momento "antes", el promedio obtenido de las mediciones 3 y 4 se denominó el momento "durante" y el estimado de las mediciones 5 y 6 el momento "después”. El impacto de la intervención se estudió para las frutas y verduras de manera independiente en el grupo intervenido y control. La evaluación del impacto de cada una de las dos estrategias se estudio de dos maneras; a) calculando la diferencia entre e intra grupos como lo sugiere Bland y Alman (24), estas diferencias fueron estimadas entre la primera y última medición y b) estimando el comportamiento del consumo en el tiempo en los momentos "antes", "durante" y "después". El impacto también fue evaluado estimando el número de hogares que consumían cinco o más porciones de FV en la línea de base y post intervención.

La estrategia de intervención y los procedimientos utilizados fueron avalados por el comité de ética en salud de la Universidad Industrial de Santander. Todos los participantes firmaron un consentimiento informado como lo establecen los principios éticos para las investigaciones médicas en seres humanos de la Declaración de Helsinki. Toda la información recolectada fue digitada por duplicado y validada para descartar errores en EpiInfo versión 6,04 (25). Los procesos estadísticos fueron realizados con Stata/SE, versión 10,1 (26).

\section{RESULTADOS}

Dieciséis hogares fueron intervenidos con la estrategia A. Veintitrés hogares fueron intervenidos con la estrategia B. Treinta y cuatro hogares sirvieron como controles.

\section{Características de la población}

En la intervención A. En el 75\% de los hogares el jefe es el padre, el trabajo es permanente en 10 de los 12 sujetos que manifestaron tenerlo. El 50\% de los hogares manifestó contar con dinero o medios necesarios para conseguir los alimentos. Dos hogares (12,5\%) recibían ayuda alimentaria de programas estatales. Seis hogares $(37,5 \%)$ consumían frutas diariamente, en nueve hogares $(56,2 \%)$ se consideró que no consumían frutas en suficiente cantidad para que sus miembros estuvieran saludables, en ocho de estos nueve $(88,9 \%)$ se argumentó que la causa era el costo de las mismas. Las frutas se compraban $75 \%$ de las veces en la plaza, el $25 \%$ restante en el supermercado, se compran con una frecuencia semanal en $43,7 \%$ y quincenal en $18,7 \%$ de los hogares. En nueve hogares $(56,2 \%)$ se consumen verduras diariamente, en diez hogares $(62,5 \%)$ se consideró que no se consumían verduras en suficiente cantidad para que sus miembros estuvieran saludables, en cinco hogares de ellos $(50,0 \%)$ la razón argumentada es que no se conseguían y en dos de ellos $(20,0 \%)$ su elevado costo. Las verduras se compraban $81,3 \%$ de las veces en la plaza, el $18,7 \%$ restante en el supermercado, se compraban con una frecuencia semanal en el 62,5\% y diaria en $18,7 \%$ de los hogares. El consumo de cinco porciones diarias de FV no se observó en este grupo de hogares. El 100\% de los responsables de preparar el menú familiar manifestó su gusto por las frutas y el $93,7 \%$ por las verduras.

En la intervención B. En el 74\% de los hogares el jefe es el padre, el trabajo es permanente en 9 de los 10 sujetos que manifestaron tenerlo. El 73,9\% de los hogares manifestaron contar con dinero o medios necesarios para conseguir los alimentos. Cinco hoga- 
res $(21,7 \%)$ recibían ayuda alimentaria de programas estatales. Nueve hogares $(39,1 \%)$ se consumen frutas diariamente En doce hogares $(52,2 \%)$ se consideró que no se consumían frutas en suficiente cantidad para que sus miembros estuvieran saludables, en siete de los doce $(53,8 \%)$ se argumentó que la causa era el costo de las mismas. Las frutas se compraban $43,5 \%$ de las veces en la plaza, y $39,1 \%$ en el supermercado, se compraban con una frecuencia semanal en $43,7 \%$ y quincenal en el $13,0 \%$ de los hogares. Dieciséis hogares $(69,6 \%)$ consumían verduras diariamente, en seis hogares $(26,1 \%)$ se consideró que no consumían verduras en suficiente cantidad para que sus miembros estuvieran saludables, en cuatro hogares $(66,7 \%)$ la razón argumentada era que no les gustan y en uno de ellos $(16,7 \%)$ su elevado costo. Las verduras se compraban en $60,9 \%$ de las veces en la plaza, $26,1 \%$ en el supermercado y el $13,0 \%$ restante en la tienda: se compraban con una frecuencia semanal en $43,5 \%$ y diaria en $34,8 \%$ de los hogares. El consumo de cinco porciones diarias de FV se presentaba en el 4,3\% de los hogares. El 87,0\% de los responsables de preparar el menú familiar manifestó su gusto por las frutas y el $95,7 \%$ por las verduras.

Grupo control. En el 73,5\% de los hogares el jefe es el padre, el trabajo era permanente en 8 de los 14 sujetos que manifestaron tenerlo. El $64,7 \%$ de los hogares manifestó contar con dinero o medios necesarios para conseguir los alimentos. Un hogar $(2,9 \%)$ recibía ayuda alimentaria de programas estatales. En dieciséis hogares $(47,1 \%)$ se consumían frutas diariamente, en 17 hogares $(50,0 \%)$ se consideró que no se consumían frutas en suficiente cantidad para que sus miembros estuvieran saludables, en diez de ellos $(58,8 \%)$ se argumentó que la causa era el costo de las mismas. Las frutas se compraban $61,8 \%$ de las veces en la plaza, $17,6 \%$ en el supermercado, se compraban con una frecuencia semanal en $32,5 \%$ y quincenal en $5,9 \%$ de los hogares. En dieciocho hogares $(52,9 \%)$ se consumían verduras diariamente, en quince hogares $(44,1 \%)$ se consideró que no se consumían verduras en suficiente cantidad para que sus miembros estuvieran saludables, en seis hogares de ellos $(40,0 \%)$ la razón argumentada es que no les gustaban y en cuatro de ellos $(26,7 \%)$ su elevado costo. Las verduras se compraban en $70,6 \%$ de las veces en la plaza, $14,7 \%$ en el supermercado y $8,9 \%$ en la tienda, se compran con una frecuencia semanal en el $44,1 \%$ y diaria en $29,48 \%$ de los hogares. El consumo de cinco porciones diarias de FV se presenta en el $2.0 \%$

\section{CUADRO 1}

Características de la población estudiada.

\begin{tabular}{|c|c|c|c|c|c|c|}
\hline \multirow[b]{2}{*}{ Variable } & \multicolumn{2}{|c|}{ Estrategia A } & \multirow{2}{*}{$\begin{array}{c}\text { Valor } \\
\mathbf{p}\end{array}$} & \multicolumn{2}{|c|}{ Estrategia B } & \multirow{2}{*}{$\begin{array}{c}\text { Valor } \\
\mathbf{p}\end{array}$} \\
\hline & $\begin{array}{l}\text { Intervenido } \\
\text { [16] }\end{array}$ & $\begin{array}{l}\text { Control } \\
{[34]}\end{array}$ & & $\begin{array}{l}\text { Intervenido } \\
{[23]}\end{array}$ & $\begin{array}{l}\text { Control } \\
{[34]}\end{array}$ & \\
\hline Escolaridad madre (años) & $7,6(5,9$ a 9,3$) *$ & $6,5(5,3$ a 7,6$)$ & 0,249 & $7,3(6,0$ a 8,7$)$ & $6,5(5,3$ a 7,6$)$ & 0,319 \\
\hline Escolaridad jefe hogar (años) & $5,8(3,2$ a 8,4$)$ & $6,9(5,5$ a 8,3$)$ & 0,434 & $7,3(5,8$ a 8,7$)$ & $6,9(5,5$ a 8,3$)$ & 0,730 \\
\hline Miembros en la familia & $5,1(4,2$ a 6,0$)$ & $4,8(4,3$ a 5,4$)$ & 0,579 & $5,3(4,8$ a 5,8$)$ & $4,8(4,3$ a 5,4$)$ & 0,245 \\
\hline Menores de siete años & $1,1(0,5$ a 1,6$)$ & $0,9(0,6$ a 1,2$)$ & 0,577 & $1,3(1,0$ a 1,7$)$ & $0,9(0,6$ a 1,2$)$ & 0,045 \\
\hline \multicolumn{7}{|l|}{ Trabaja la madre } \\
\hline $\mathrm{Si}$ & $9\{56,2\} \dagger$ & $14\{41,2\}$ & 0,518 & $10\{43,5\}$ & $14\{41,2\}$ & 0,707 \\
\hline Estrato socioeconómico & & & 0,432 & & & 0,982 \\
\hline 1 & $4\{25,0\}$ & $4\{11,8\}$ & & $3\{13,0\}$ & $4\{11,8\}$ & \\
\hline 2 & $9\{56,2\}$ & $20\{58,8\}$ & & $13\{56,5\}$ & $20\{58,8\}$ & \\
\hline 3 & $3\{18,8\}$ & $10\{29,4\}$ & & $7\{30,5\}$ & $10\{29,4\}$ & \\
\hline \multicolumn{7}{|l|}{ ¿Consumen frutas diariamente? } \\
\hline $\mathrm{Si}$ & $6\{37,5\}$ & $16\{47,1\}$ & 0,525 & $9\{39,1\}$ & $16\{47,1\}$ & 0,554 \\
\hline \multicolumn{7}{|l|}{ ¿Consumen verduras diariamente? } \\
\hline $\mathrm{Si}$ & $9\{56,2\}$ & $18\{52,9\}$ & 0,827 & $16\{69,6\}$ & $18\{52,9\}$ & 0,159 \\
\hline
\end{tabular}

Promedio e intervalo de confianza del $95 \%$. † Número y porcentaje. 
de los hogares. El 100\% de los responsables de preparar el menú familiar manifestó su gusto por las frutas y el $79,4 \%$ por las verduras.

Con excepción de la variable ayuda alimentaria en la estrategia $B(p=0,023)$, ninguna variables es diferente estadísticamente entre los hogares intervenidos y control. El cuadro 1 presenta otras características de la población estudiada.

\section{Evaluación de impacto}

Grupo control. El consumo medio de porciones/ día de frutas en la línea de base fue de 1,49 (IC; 1,26 a 1,72), en la última medición de 1,34 (IC; 1,16 a 1,52). La media de la frecuencia/mes del consumo en frutas en la línea de base fue de 6,09 (IC; 5,24 a 6,94), en la última medición de 5,10 (IC; 4,71a 5,50). El consumo medio de porciones/día de verduras en la línea de base fue de 0,49 (IC; 0,31 a 0,67), en la última medición de 0,80 (IC; 0,63 a 0,96). La media de la frecuencia/mes del consumo de verduras en la línea de base fue de 7,37 (IC; 6,35 a 8,39), en la última medición de 6,15 (IC; 5,47 a 6,84$)$. La combinación de frutas y verduras también fue estudiada, el consumo medio de porciones/día de frutas y verduras en la línea de base para el grupo control fue de 2,00 (IC; 1,75 a 2,25), en la última medición de 2,15 (IC; 1,92 a 2,38). La media de la frecuencia/mes del consumo de frutas y verduras en la línea de base fue de 6,71 (IC; 5,94 a 7,49), en la última medición de 5,61 (IC; 5,16 a 6,07).

Estrategia A. El consumo medio de porciones/ día de frutas en la línea de base fue de 1,43 (IC; 1,12 a 1,73), en la última medición de 1,91 (IC; 1,58 a 2,23). La media de la frecuencia/mes del consumo de frutas en la línea de base fue de 5,51 (IC; 3,90 a 7,12), en la última medición de 5,25 (IC; 4,62 a 5,88). El consumo medio de porciones/día de verduras en la línea de base fue de 0,39 (IC; 0,13 a 0,66), en la última medición de $1,00$ (IC; 0,75 a 1,24$)$. La media de la frecuencia/mes del consumo de verduras en la línea de base fue de 5,41 (IC; 4,34 a 6,48), en la última medición de 4,47 (IC; $3,79$ a 5,14$)$.

El consumo medio de porciones/día de frutas y verduras en la línea de base fue de 1,82 (IC; 1,47 a 2,17), en la última medición de 2,91 (IC; 2,42 a 3,39). La media de la frecuencia/mes del consumo de frutas y verduras en la línea de base fue de 5,46 (IC; 4,31 a 6,61), en la

\section{CUADRO 2}

\section{Diferencia entre porciones/día y frecuencia de consumo/mes; (después-antes), en dos estrategias de intervención para aumentar el consumo de frutas y verduras.}

\begin{tabular}{|c|c|c|c|c|c|c|c|c|}
\hline \multirow{4}{*}{ Intra grupos } & \multicolumn{8}{|c|}{ ESTRATEGIA A } \\
\hline & \multicolumn{4}{|c|}{ Frutas } & \multicolumn{4}{|c|}{ Verduras } \\
\hline & $\mathrm{C}_{\mathrm{D}}-\mathrm{C}_{\mathrm{A}} *$ & $\mathrm{C}_{\mathrm{D}^{-}}-\mathrm{C}_{\mathrm{A}} \dagger$ & $\mathrm{I}_{\mathrm{D}}-\mathrm{I}_{\mathrm{A}} *$ & $\mathrm{I}_{\mathrm{D}}-\mathrm{I}_{\mathrm{A}} \dagger$ & $\mathrm{C}_{\mathrm{D}}-\mathrm{C}_{\mathrm{A}} *$ & $\mathrm{C}_{\mathrm{D}}-\mathrm{C}_{\mathrm{A}} \dagger$ & $\mathrm{I}_{\mathrm{D}}-\mathrm{I}_{\mathrm{A}} *$ & $\mathrm{I}_{\mathrm{D}}-\mathrm{I}_{\mathrm{A}} \dagger$ \\
\hline & $-0.15 \ddagger$ & $-0.99 \div$ & $0.48 \div$ & -0.26 & $0.31 \div$ & $-1.22 \div$ & $0.61 \ddagger$ & -0.94 \\
\hline \multicolumn{9}{|l|}{ Entre grupos } \\
\hline & $\begin{array}{c}\mathrm{I}_{\mathrm{A}}-\mathrm{C}_{\mathrm{A}} * \\
-0.06\end{array}$ & $\begin{array}{c}\mathrm{I}_{\mathrm{A}}-\mathrm{C}_{\mathrm{A}} \dagger \\
-0.58\end{array}$ & $\begin{array}{c}\mathrm{I}_{\mathrm{D}}-\mathrm{C}_{\mathrm{D}} * \\
0.57 \ddagger\end{array}$ & $\begin{array}{c}\mathrm{I}_{\mathrm{D}}-\mathrm{C}_{\mathrm{D}} \dagger \\
0.15\end{array}$ & $\begin{array}{c}\mathrm{I}_{\mathrm{A}}-\mathrm{C}_{\mathrm{A}} * \\
-0.10\end{array}$ & $\begin{array}{l}\mathrm{I}_{\mathrm{A}}-\mathrm{C}_{\mathrm{A}} \dagger \\
-1.96 \doteqdot\end{array}$ & $\begin{array}{c}\mathrm{I}_{\mathrm{D}}-\mathrm{C}_{\mathrm{D}} * \\
0.20\end{array}$ & $\begin{array}{l}\mathrm{I}_{\mathrm{D}}-\mathrm{C}_{\mathrm{D}} \dagger \\
-1.68 \dagger\end{array}$ \\
\hline & \multicolumn{8}{|c|}{ ESTRATEGIA B } \\
\hline & \multicolumn{4}{|c|}{ Frutas } & \multicolumn{4}{|c|}{ Verduras } \\
\hline Intra grupos & & & & & & & & \\
\hline & $\mathrm{C}_{\mathrm{D}}-\mathrm{C}_{\mathrm{A}} *$ & $\mathrm{C}_{\mathrm{D}}-\mathrm{C}_{\mathrm{A}} \dagger$ & $\mathrm{I}_{\mathrm{D}}-\mathrm{I}_{\mathrm{A}} *$ & $\mathrm{I}_{\mathrm{D}}-\mathrm{I}_{\mathrm{A}} \dagger$ & $\mathrm{C}_{\mathrm{D}}-\mathrm{C}_{\mathrm{A}} *$ & $\mathrm{C}_{\mathrm{D}}-\mathrm{C}_{\mathrm{A}} \dagger$ & $\mathrm{I}_{\mathrm{D}}-\mathrm{I}_{\mathrm{A}} *$ & $\mathrm{I}_{\mathrm{D}}-\mathrm{I}_{\mathrm{A}} \dagger$ \\
\hline & $-0.15 \ddagger$ & $-0.99 \div$ & 0.25 & $-1.39 \ddagger$ & $0.31 \div$ & $-1.22 \div$ & 0.01 & $-1.06 \div$ \\
\hline \multirow[t]{2}{*}{ Entre grupos } & & & & & & & & \\
\hline & $\begin{array}{c}\mathrm{I}_{\mathrm{A}}-\mathrm{C}_{\mathrm{A}} * \\
0.07\end{array}$ & $\begin{array}{c}\mathrm{I}_{\mathrm{A}}-\mathrm{C}_{\mathrm{A}} \dagger \\
-070\end{array}$ & $\begin{array}{c}\mathrm{I}_{\mathrm{D}}-\mathrm{C}_{\mathrm{D}} * \\
0.47+\end{array}$ & $\mathrm{I}_{\mathrm{D}}-\mathrm{C}_{\mathrm{D}} \dagger$ & $\mathrm{I}_{\mathrm{A}}-\mathrm{C}_{\mathrm{A}} *$ & $\begin{array}{l}\mathrm{I}_{\mathrm{A}}-\mathrm{C}_{\mathrm{A}} \dagger \\
-146 \dagger\end{array}$ & $\begin{array}{c}\mathrm{I}_{\mathrm{D}}-\mathrm{C}_{\mathrm{D}} * \\
0.06\end{array}$ & $\begin{array}{l}\mathrm{I}_{\mathrm{D}}-\mathrm{C}_{\mathrm{D}} \dagger \\
-129+\end{array}$ \\
\hline & & & 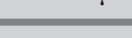 & 0.00 & & & & \\
\hline $\begin{array}{l}C_{D} \text {; Control desp } \\
\text { Antes hace refere } \\
\dagger \text { Para la frecuen } \\
* \text { Para el número } \\
\dagger \text { Para la frecuen }\end{array}$ & $\begin{array}{l}\text {; Control a } \\
\text { la medida e } \\
\text { dia de consı } \\
\text { ciones día } \\
\text { dia de consı }\end{array}$ & $\begin{array}{l}\mathrm{I}_{\mathrm{D}} \text {; Interve } \\
\text { línea de bi } \\
\text { al mes, pr } \\
\text { vado de un }\end{array}$ & $\begin{array}{l}\text { después, I } \\
\text { Para el nı } \\
\text { lio acumul } \\
\text { H. }\end{array}$ & $\begin{array}{l}\text { ntervenido } \\
\text { ero de porc } \\
\text { de } 18 \text { frut }\end{array}$ & $\begin{array}{l}\text { (Después } \\
\text { día derivade } \\
17 \text { verdura } \\
17 \text { verdur }\end{array}$ & $\begin{array}{l}\text { lace referenci } \\
\text { de un } \mathrm{R} 24 \mathrm{H} \\
\text { s. } \neq \mathrm{p}<0.05 \text {. } \\
\text { as. } \ddagger \mathrm{p}<0.05 \text {. }\end{array}$ & la medición & \\
\hline
\end{tabular}


última medición de 4,87 (IC; 4,36 a 5,37).

Estrategia B. El consumo medio de porciones/ día de frutas en la línea de base fue de 1,56 (IC; 1,33 a 1,80 ), en la última medición de 1,81 (IC; 1,57 a 2,06). La media de la frecuencia/mes del consumo de frutas en la línea de base fue de 6,79 (IC; 5,84 a 7,74), en la última medición de 5,40 (IC; 4,84 a 5,97). El consumo medio de porciones/día de verduras en la línea de base fue de 0,85 (IC; 0,55 a 1,14), en la última medición de 0,86 (IC; 0,60 a 1,12). La media de la frecuencia/mes del consumo de verduras en la línea de base fue de 5,92 (IC; 5,14 a 6,69), en la última medición de 4,86 (IC; $4,33$ a 5,39$)$.

El consumo medio de porciones/día de frutas y verduras en la línea de base fue de 2,41 (IC; 2,02 a 2,80), en la última medición de 2,67 (IC; 2,31 a 3,04). La media de la frecuencia/mes del consumo de frutas y verduras en la línea de base fue de 6,37 (IC; 5,54 a 7,19), en la última medición de 5,14 (IC; 4,66 a 5,62).

Otra forma de evaluar el impacto fue establecer el número de hogares que en las líneas de base y post intervención consumían cinco o más porciones de $\mathrm{FV}$ al día. En los intervenidos el 3,0\% en la línea de base consumían cinco o más porciones, al finalizar las estrategias aumentaron al 26,3\%, un incremento de 8,8 veces. En el grupo control al inicio este consumo recomendado de cinco o más porciones/día fue de $2,0 \%$, al final de $0,0 \%$. $\mathrm{El}$ aumento no fue diferencial dada la estrategia de inter- vención, en la estrategia A paso del $0 \%$ de hogares en la línea de base al 23,5\%, en la B del 4,3 al 28,6\%.

El cuadro 2 presenta las diferencias medias intragrupo y entregrupos para $\mathrm{FV}$ en las dos estrategias. Las figuras 1 y 2 presentan el efecto de las diferentes estrategias sobre el consumo de FV en tres diferentes momentos del tiempo; antes o línea base, durante y después o final de la intervención. El cuadro 3 presenta el resumen del efecto de las dos estrategias desagregado por el consumo de porciones/día y la frecuencia de consumo/mes. En las figuras 1-a y 1-b se nota claramente el aumento sobre el consumo de porciones/día para las frutas.

\section{DISCUSIÓN}

Las estrategias desarrolladas estuvieron dirigidas a familias con niños escolarizados, por ser el entorno social y de más influencia en la formación de hábitos alimentarios (27) y porque su participación es trascendental cuando se busca promocionar hábitos en los preescolares y escolares (17). La participación familiar debe iniciarse desde el momento de la planificación, esto se logró con la metodología cualitativa utilizada en la formulación, seguimiento y evaluación de las estrategias implementadas, de acuerdo a la recomendación de expertos de complementar la investigación cualitativa y cuantitativa en todas las etapas de la intervención (21).

Impacto alcanzado. La última medición sobre consumo de FV se realizó dos meses después de cul-

\section{FIGURA 1}

\section{Consumo de frutas}
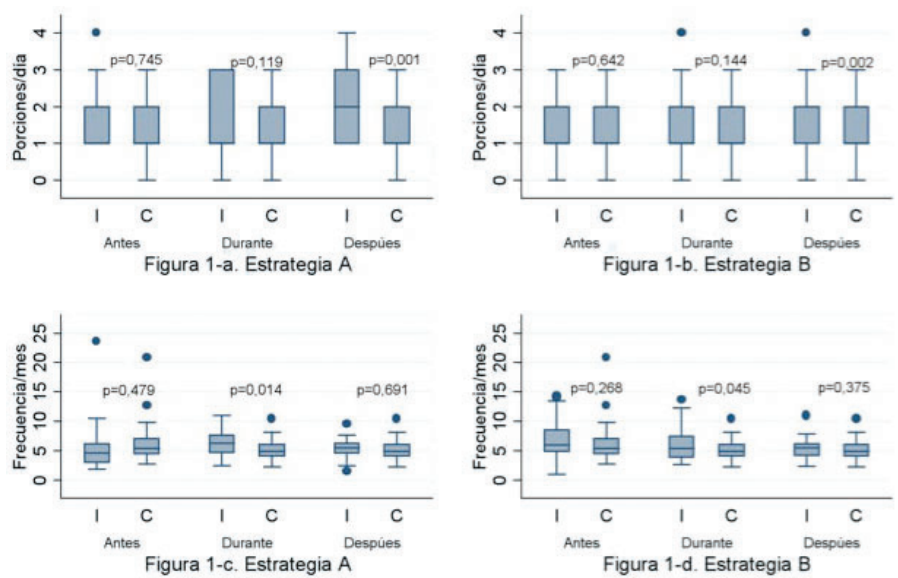

I: Intervenido; C: Control 
minar las estrategias, lo que permite aproximarse a la sostenibilidad de la intervención. Sin embargo, es aconsejable para una futura experiencia, realizar una evaluación de sostenibilidad en un tiempo mayor. Las dos intervenciones permitieron incrementar el consumo de $\mathrm{FV}$ en proporciones mayores a las reportadas en otros estudios.

Un estudio con escolares realizado durante 7 años, logró un incremento para $\mathrm{FV}$ de 0,26 porciones/día en niñas, pero no en niños (28), similar al reportado en la estrategia B. Otro estudio realizado por la Universidad de Harvard, mostró que después de 19 meses se logró aumentar el consumo de FV en escolares en 0,36 porciones/día (IC; 0,10 a 0,62) (29). Después de dos años de intervención del programa "cinco al día", estudio realizado por la Universidad de Maryland, la ingesta de FV aumentó en 0,56 porciones/día (30). Un estudio realizado en población similar a la estudiada mostró que después de 10 meses se incrementó el consumo de frutas en 1,3 veces por semana, pero no en el consumo de verduras (18).

Las estrategias fueron ajustadas a la recomendación de un mínimo de 10 sesiones para obtener cambios positivos sobre el consumo de FV (31), y tenían como objetivo mejorar los conocimientos nutricionales relacionados con las $\mathrm{FV}$, aspecto que contribuye a lograr impacto en las intervenciones con FV (9). Las estrategias incluyeron degustación de alimentos, aspecto favorito para escolares y adultos $(8,9)$. La estrategia A logró el doble del impacto en el consumo de frutas que la B -0,5 porciones versus $0,25-$, y fue la única que impactó el consumo de verduras en proporciones mayores que las alcanzadas en otros estudios - de 0,39 al inicio a 1,00 porciones al final-.

Estos resultados obedecen a que se desarrolló la autoeficacia en la preparación y planeación del menú en los responsables directos de la preparación de alimentos. Como lo sugerido en otras intervenciones se propició una participación activa mediante el diálogo de saberes para aclarar dudas y enriquecerse mutuamente con la experiencia del otro, aspectos determinantes de la calidad y sostenibilidad del aprendizaje social $(8,9,32)$. Además, se realizó un seguimiento personalizado mediante visitas domiciliarias. En la estrategia B, todas las sesiones se realizaron con los niños y sólo se incluyeron tres encuentros con los responsables de la preparación de alimentos, sin incluir talleres de preparación, las temáticas para ambos grupos fueron similares.

Ámbito escolar. Las instituciones educativas de enseñanza básica son importantes para implementar estrategias de promoción de la salud, cubren por largos periodos de tiempo y de manera sostenida amplios grupos de población (33). La promoción de una alimentación saludable debe hacerse desde edades tempranas, por ser la de mayor receptividad hacia el consumo de nuevos alimentos (8), involucrar a la población escolar

\section{FIGURA 2}

\section{Consumo de verduras}
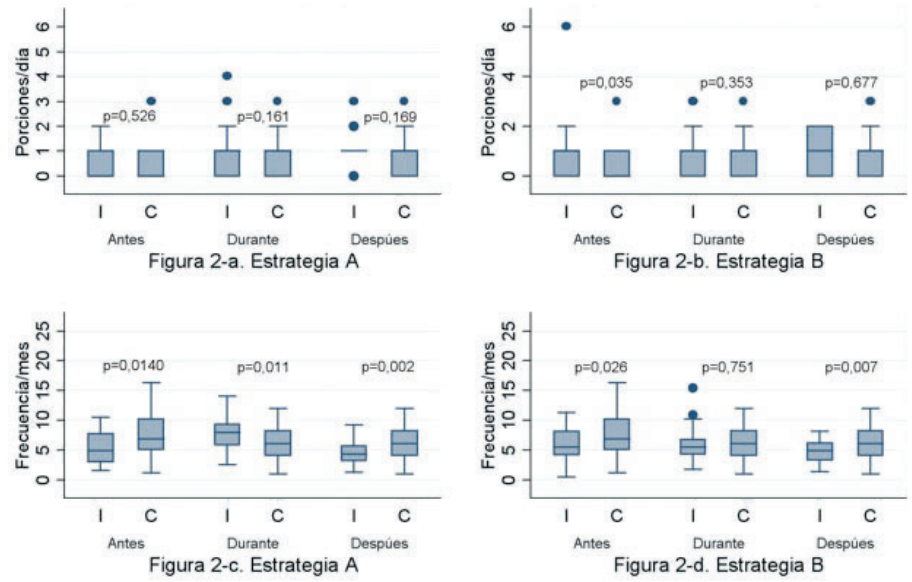
permite aprovechar la influencia que tienen los niños en las actitudes y comportamientos de sus padres (34), en el presente estudio los padres reconocieron a sus hijos como agentes motivadores hacia el incremento del consumo de FV en las familias. En el proyecto participaron los docentes, ajustando los contenidos de los mensajes al desarrollo cognitivo del niño (35) y las familias por ser un factor exitoso de estas intervenciones con este grupo de población (36).

Participación de los involucrados. Un reto de las intervenciones comunitarias es mantener la asistencia durante la ejecución del programa (32). Algunos estudios ofrecen una retribución económica por la participación en intervenciones. La participación se mejora cuando se trabaja con población cautiva y cuando se aplicaran métodos flexibles para que participen a distancia, estas características corresponden a la Estrategia B, por lo cual la participación se mantuvo. En la estrategia A, la participación estuvo condicionada únicamente al interés de los participantes, a pesar de que el tiempo de ejecución fue de ocho meses y que no existió ningún tipo de retribución, la participación se logró mantener hasta el final de la intervención en un 50\% de los asistentes iniciales.

A manera de conclusión puede afirmarse que; 1) Los resultados de impacto logrados en el presente estudio superan lo reportado en otras investigaciones. Algunos factores que incidieron en los resultados fueron, a) involucrar los participantes en la identificación de las barreras y las posibles soluciones b) favorecer la participación activa de todos los involucrados, c) utilizar metodologías cualitativas y cuantitativas para el diseño, el seguimiento y la evaluación de la intervención, d) utilizar las lecciones aprendidas por el grupo investigador. 2) Para medir el impacto en el consumo de FV, es más apropiado cuantificar el consumo en términos de porciones/día, más que en el número de veces/día, dado que permite precisar de mejor forma la cantidad consumida

\section{CUADRO 3}

\section{Resumen del efecto alcanzado en dos estrategias de intervención para aumentar el consumo de frutas y verduras.}

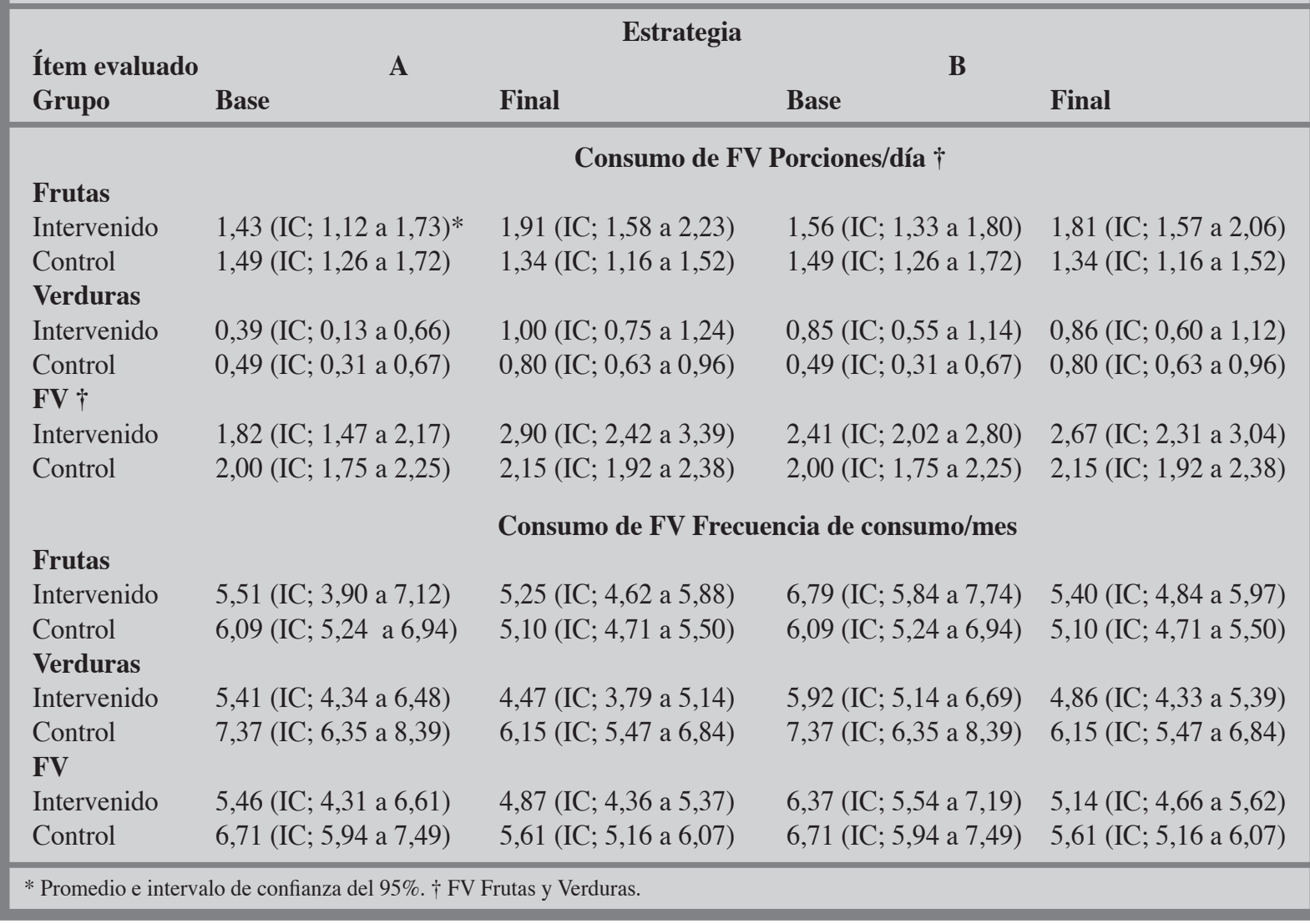


y ofrece un mejor indicador para realizar seguimiento y evaluación de la política mundial de promoción del consumo de FV.

Los hallazgos permiten recomendar las dos estrategias, la estrategia A, logra impactar positivamente el consumo de frutas y verduras, pero a diferencia de la estrategia B, requiere mayor seguimiento por los coordinadores, y presencia y permanencia de los participantes. La estrategia B sólo impactó el consumo de frutas, sin embargo requiere de menor logística y presencia de los participantes y permite alcanza una mayor cobertura. Para esta estrategia se recomienda ampliar el tiempo de aplicación para impactar el consumo de verduras. Es necesario resaltar que no fue objeto de este estudio evaluar la sostenibilidad de los resultados en el tiempo.

\section{RESUMEN}

Durante los años 2007-2008 se realizó en Lebrija, Colombia, un estudio para evaluar el impacto de dos estrategias implementadas en familias de niños escolarizados, que tuvieron como objetivo incrementar el consumo de frutas y verduras en el hogar. Dieciséis hogares fueron intervenidos con la estrategia A. Veintitrés hogares fueron intervenidos con la estrategia B. Treinta y cuatro hogares sirvieron como controles. Las estrategias incluyeron entre otros, talleres informativos, preparación de recetas, actividades grupales y evaluación de tareas. En los hogares intervenidos el 3,0\% en la línea de base consumían cinco o más porciones, al finalizar las estrategias aumentaron al 26,3\%, un incremento de 8,8 veces. En el grupo control al inicio este consumo recomendado de cinco o más porciones/día fue de 2,0\%, al final de $0,0 \%$. El aumento no fue diferencial dada la estrategia de intervención, en la estrategia A paso del $0 \%$ de hogares en la línea de base al $23,5 \%$, en la B del 4,3 al 28,6\%. Las dos estrategias evaluadas tuvieron impacto positivo, la A sobre el consumo de frutas y verduras, la B sobre el consumo de frutas.

Palabras clave: evaluación de impacto; frutas; verduras; ensayo controlado aleatorio; estudios de intervención; Colombia.

Dirigir la correspondencia a:

Profesora

Gloria Esperanza Prada Gómez

Carrera 32 No. 29-31

Facultad de Salud

CIE. Tercer Piso

Universidad Industrial de Santander

Bucaramanga

Colombia.

Sur América
Tele fax; (57-7) 6345781 / 6323215

e-mail: gprada@uis.edu.co

gprada@telebucaramanga.net.co

Declaración de conflicto de interés: Los autores declaran que no tienen conflicto de interés de ningún tipo, ni real o potencial sobre los resultados presentados.

\section{BIBLIOGRAFÍA}

1. World Health Organization (WHO). Global strategy on diet, physical activity and health. Mayo 2002. (Fecha de acceso octubre 15 de 2005). URL disponible en http://www.who.int/dietphysicalactivity/en/.

2. Havas S, Heimenchnger J, Reynolds K, et al. 5 a day for better health: a new research initiative. J Am Diet Assoc 1994;94:32-36.

3. Boutayeb A, Boutayeb S. The burden of non communicable diseases in developing countries. Int $\mathbf{J}$ Equity Health 2005; 14: 4-2.

4. World Health Organization (WHO). Report of a join WHO/FAO expert consultation. Diet, Nutritition and the prevention of chronic diseases. WHO Technical Report series 916, Geneva, 2003.

5. Williams C. Healthy eating: clarifying advice on fruit and vegetable consumption. BMJ 1995; 310:1453-1455.

6. Instituto Colombiano de Bienestar Familiar (ICBF). Encuesta Nacional de la Situación Nutricional en Colombia, 2005. Bogotá, Colombia, 2005.

7. Anderson AS, Cox DN et al. (1998). Take Five, a nutrition education intervention to increase fruit and vegetable intakes: impact on attitudes towards dietary change. British J Nutr 80; (2): 133-140.

8. Prada GE, Gamboa EM, Jaime ML. Representaciones sociales sobre alimentación saludable en población vulnerable. Bucaramanga, Santander. Colombia. Salud UIS 2006; 38(3):181-188

9. Cullen KW, Bartholomew LL, Parcel GS. Girl scouting: An effective channel for nutrition education. $J$ Nutr Educ 1997; 29: 86-91.

10. Domel SB, Baranowski T, Davis H, et al. Development and evaluation of a school intervention to increase fruit and vegetable consumption among 4th and 5th grade students. J Nutr Educ 1993;25:345349.

11. Perry CL, Mullis RM, Maile MC. Modifying the eating behavior of children. J Sch Health 1985; 55: 399-402.

12. Graves K, Shannon B, Sims L,Johnson L. Nutrition knowledge and attitudes of elementary school students after receiving nutrition education. J Am 
Diet Assoc 1982;81:422-427.

13. Shannon B, Chen AN.A three-year school-based nutrition education study. J Nutr Educ 1988; 20:114124.

14. Constante P, Sarti FM, Faria M, Monteiro CA. Impacto de una intervención basada en la comunidad, en el mayor consumo de frutas y vegetales en familias de bajos ingresos, Sao Paulo, Brasil. Rev Chil Nutr 2006; 33 (Supl. 1): 266-271.

15. OPS, OMS. Estrategia regional y plan de acción para un enfoque integrado sobre la prevención y el control de las enfermedades crónicas. Washington, D.C: OPS. 2007. Disponible en; http://www.paho. org/Spanish/AD/DPC/NC/reg-strat-cncds.pdf. Consultado en abril 30 de 2009.

16. Perry C, Bishop D, Taylor G. Changing fruit and vegetables consumption among children. The 5-aDay Power Plus Program in St Paul, Minnesota. Am J Public Health 1998;88:603-609.

17. Serra L, Aranceta J. Nutrición y Salud Pública: Métodos, bases científicas y aplicaciones. Barcelona: Masson, 2006. pp 848.

18. Prada GE, Dubeibe LY, Herrán OF, Herrera M. Evaluación del impacto de un ensayo comunitario sobre el consumo de frutas y verduras en Colombia. Salud Pública Méx 2007;49 (1):11-19.

19. Herrán OF, Bautista LE. Calidad de la dieta en Bucaramanga. Colombia, 1998-2003. I. Patrón alimentario en 1998. Salud UIS 2003;35:63-70.

20. Herrán OF, Bautista LE. Calidad de la dieta de la población adulta en Bucaramanga y su patrón alimentario. Colomb Med 2005;36:94-102.

21. Pelto GH. Anthropological contributions to nutrition education research. JNE 1981;13 (1 Suppl 1): S2-813.

22. Soto A, Prada GE, Dubeibe LY. Manual de preparaciones con hortalizas, verduras y leguminosas verdes. UIS-ICBF. División de Publicaciones. UIS. Bucaramanga. 2002.

23. Soto A, Prada GE, Gómez L. Con hortalizas y verduras, sabor y nutrición. Bucaramanga. 2006. UIS. Publicaciones Publicom PDC. ISBN 958-338760-6.

24. Bland JM, Altman DG. Measuring agreement in method comparison studies. Stat Methods Med Res 1999; 8:135-160.

25. CDC. EpiInfo, versión 6.04d. Epidemiología en ordenadores. Atlanta, Georgia. Enero, 2001.

26. StataCorp. Stata Statistical Software: Release 10.1 Collegue Station, TX: StataCorp LP. 2008.

27. Aldinger CE, Jones JT. Healthy Nutrition. An essential element of a Health-Promoting School. World Health Organization Information Series on School Health. Document four. Geneve: World Health Organization. 1998.

28. Perry C, Luepker R, Murray D. Kurth C. Parent involvement with Children's Health Promotions: The Minnesota Home Team. Am J Public Health 1998;78:1156-1160.

29. Gortmaker S, Cheung L, Peterson K, Chomitz G. Impact of a school-based interdisciplinary intervention on diet and physical activity among urban primary school children. Arch Pediatr Adolesc Med 1999;153:975-983.

30. Havas S, Anliker J, Damron D. Final results of the Maryland WIC 5-a- Day Promotion Program. Am J Public Health 1998;88:1161-1167.

31. Howison D, Neidermyer FS, Shortridge R. Field testing a fifth-grade nutrition education program designed to change food-selection behavior. J Nutr Educ 1988;20:82-6.

32. Suazo G, Caro P, Aguirre F, Jerez C. Los talleres aprendiendo juntos. Una experiencia preventiva en el ámbito escolar. CIPPAL. Centro de investigación y difusión poblacional. Viña del Mar. Chile. 1994. Disponible en: http://redalyc.uaemex.mx/redalyc/ src/inicio/ArtPdfRed.jsp?iCve=19500202. Consultado en abril 30 de 2009.

33. Olivares S, Snel J, McGrann M, Glasauer P. Educación en nutrición en las escuelas primarias: realidad actual, necesidades y limitaciones. Food Nutr Agr 1998;22:57-62.

34. Perry C, Luepker R, Murray D. Kurth C. Parent involvement with Children's Health Promotions: The Minnesota Home Team. Am J Public Health 1998;78:1156-1160.

35. Dixey R. Heindl I, Loureiro I, Péres-Rodrigo C, Snel J. Warn king P. Healthy eating for young people in Europe. A school-based nutrition education guide. Copenhague: European Network of Health Promotion Schools; 1999.

36. Center for Disease Control and Prevention. Guidelines for school health programs to promote lifelong healthy eating. MMWR 1996;45 (No RR-9):1-41. 